\title{
A pseudo community outbreak of legionnaires' disease on Merseyside; implications for investigation of suspected clusters
}

\author{
C M Regan, Q Syed, K Mutton, B Wiratunga
}

\begin{abstract}
Summary
During the winter of 1995 , a space-time cluster of seven suspected cases of legionnaires' disease occurred on Merseyside, involving three neighbouring health districts. A multidisciplinary investigation team was established but failed to obtain convincing evidence of an outbreak. Investigation of the seven cases confirmed two as sporadic cases; other infections were found to explain the remaining cases that had been initially confirmed presumptive cases on the basis of false positive Legionella pneumophila type 1 urine ELISA antigen test result. The epidemiological and clinical features of the unconfirmed cases were also not characteristic of legionnaires' disease. The investigation team concluded that this was a pseudo outbreak on the basis of detailed epidemiological investigation. This incident raised the importance of a strong index of suspicion when investigating suspected outbreaks of legionnaires' disease in the community wherever the results of one test have not been supported by others.
\end{abstract}

Liverpool Health Authority, Department of Public Health, Hamilton House, 24 Pall Mall, Liverpool L3 6AL

C M Regan

Communicable Disease Surveillance Centre North West, Liverpool Public Health Laboratory, University Hospital Aintree, Liverpool Q Syed

Liverpool Public Health Laboratory, University Hospital Aintree

K Mutton

Department of Public Health, St Helens and Knowsley Health Authority, St Helens, Merseyside

B Wiratunga

Correspondence to: Dr Regan

(martyn.regan@

liverpool_ha.nwest.nhs.uk)

Accepted for publication 22 September 1999
Legionella outbreaks first became matters of particular concern where these were related to common sources within hospital and residential settings because of the combination of high attack rates in susceptibles and high fatality rate. ${ }^{1-4}$ More recently community outbreaks of legionellosis have also been recognised and have often been found to be associated with wet cooling tower systems. ${ }^{5-7}$ However, a definitive identification of a source of infection is not always possible because of the ubiquitous nature of legionella organisms in the environment and a low attack rate in the community. ${ }^{7-9}$ We describe an investigation of a suspected community outbreak of legionellosis that illustrates some of the practical difficulties encountered when investigating apparent clusters.

\section{The outbreak}

In January 1995, the on call public health doctor and Consultant in Communicable Disease Control (CCDC) for Liverpool District were informed about a suspected case of legionnaires' disease who had worked at a Liverpool hospital where possible nosocomial cases of legionnaires' disease have been noted in recent years (Dr R W Moyes, personal communication).

Four days later, the Liverpool Public Health Laboratory reported two further suspected cases of legionnaires' disease from Liverpool and an investigation of the case cluster was launched.

\section{The investigation}

Initial epidemiological investigations of the two new suspected cases were undertaken and, after an informal review of the epidemiological data, a formal incident team was convened to identify a possible common source of infection.

The team included CsCDC and Environmental Health Officers (EHOs) for the Merseyside health districts and local authorities, Regional Epidemiologist, Director of Liverpool Public Health Laboratory, Hospital Control of Infection Doctor and local officers of the Health and Safety Executive.

EPIDEMIOLOGICAL INVESTIGATION AND INITIAL FINDINGS

Case definition

It was agreed that the case definition for legionnaires' disease devised by the Communicable Disease Surveillance Centre $(\mathrm{CDSC})^{10}$ should be used as the basis for investigating the incident and assigning cases to the appropriate case category (fig 1: CDSC case definition). Suspected cases were interviewed either alone or jointly by the relevant CCDC and the Principal EHO concerning details of movements, buildings visited and exposures to potential sources of legionella in the 14 day period before the onset of illness in seeking to identify a common source.

Case 1 worked in the catering department of a Liverpool Hospital; movements before illness were uncertain but indicated a sedentary lifestyle apart from travel to and from her place of work. Case 2 was established to be an "odd job" man who had a variety of jobs during the two weeks before onset of illness. He had undertaken some maintenance work close to three cooling towers at a small industrial estate to the north of the city centre. Case 3 was noted to be young with an absence of risk factors for legionnaires' disease. The patient's brother had been admitted to hospital after an episode of pneumonia.

Cases 1 and 2 met the case definition for confirmed cases and case 3 fulfilled the requirements for a presumptive case on the basis of a positive Legionella pneumophila type 1 urine ELISA antigen test. There did not appear to be any clear epidemiological link between the three cases but a possible link with the city centre was noted. It was recognised by the Incident Control Team that there was known to be a community outbreak of Mycoplasma pneumoniae at this time.

A search for further cases was undertaken by informing local hospital doctors and general practitioners of the suspected outbreak and 
(i) Confirmed case:

A clinical diagnosis of pneumonia with laboratory evidence of one or more of the following:

- culture of Legionella spp from clinical specimens

- seroconversion (a fourfold rise or greater) to 64 or more by the indirect immunofluorescent antibody test (IFAT) using

$L$ pneumophila serogroup 1 yolk sac antigen

- seroconversion (a fourfold rise or greater) to 16 or more by the rapid microagglutination test (RMAT) using $L$ pneumophila serogroup 1 antigen

(ii) Presumptive case:

A clinical diagnosis of pneumonia with laboratory evidence of one or more of the following:

- a single titre of 128 or more using IFAT as above (64 or more in an outbreak)

- a single titre of 32 or more using RMAT as above

- positive urine ELISA using validated reagents*

- positive direct fluorescence (DFA) on a clinical specimen using validated monoclonal antibodies

*After this case cluster, the Legionella pneumophila type 1 urine ELISA antigen test has been classified as providing confirmatory evidence of legionnaires' disease since January 1998.

Figure 1 Case definition used by the National Surveillance Scheme for legionnaires' disease.

requesting that suspected cases of atypical pneumonia should be investigated.

Microbiological investigation

Suspected cases of atypical pneumonia were initially examined for the presence of Legionella pneumophila serogroup 1 antigen in urine, ${ }^{10-13}$ at the Central Public Health Laboratory Respiratory and Systemic Infection Laboratory, Colindale (RSIL) and serology was subsequently performed to try to demonstrate a diagnostic rise in titres to legionella antibodies using the Rapid Microagglutination Antibody test $(\text { RMAT) })^{14-15}$ and the Immunofluorescent antibody test (IFAT). ${ }^{16}$

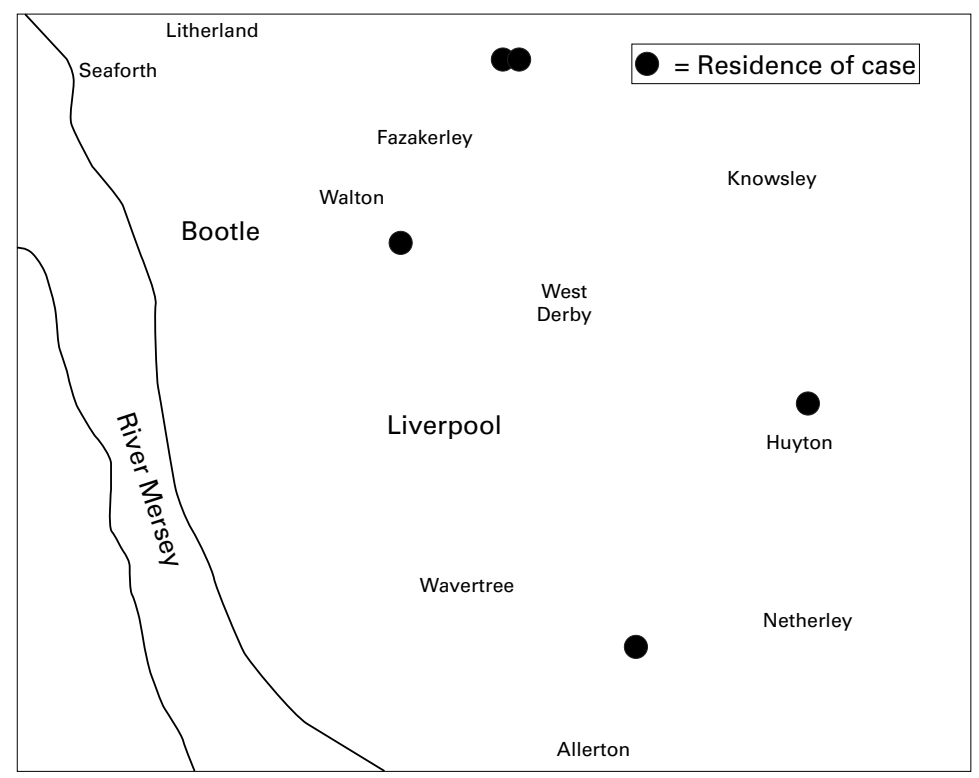

Figure 2 Geographical spread of cases.
Environmental investigation

Because of a possible link between cases and visits to the city centre during the Christmas period an initial investigation by the Liverpool City Environmental Health Department examined the operation of 11 cooling towers registered, and actively searched for potential sources and high risk plant within a half mile radius of the city centre. An investigation was also undertaken into the operation of the three cooling towers at an industrial estate close to where case 2 had been working. The maintenance log books for water systems within the Liverpool hospital where case 1 worked were reviewed to assess compliance with Health Technical Memorandum 2040. ${ }^{17}$ Water samples were also taken from the patient's home.

\section{Results of further investigations}

EPIDEMIOLOGICAL/ENVIRONMENTAL

INVESTIGATION

It was confirmed that there was no suspect plant in the vicinity of where case 1 lived and water samples taken from the patient's home for analysis were negative for legionella. Legionella pneumophila type 6 was isolated from a male shower in the same department where case 1 worked. This hospital was known to have water systems intermittently colonised by Legionella pneumophila type 6, which is an uncommon cause of legionellosis. ${ }^{18}$ No additional information was obtained concerning potential sources of exposure and she died during the investigation.

Visual inspection of the cooling towers close to where case 3 had worked was unsatisfactory, however no legionella organisms were identified in samples taken.

Of the known cooling towers in the city centre some premises had changed to dry systems but no problems were identified.

Two further presumptive cases were ascertained (cases 4 and 5); one case was reported by a hospital doctor and the other by a general practitioner; both cases were considered presumptive on the basis of positive results for the Legionella pneumophila type 1 urine ELISA antigen test. Case 5 had recently returned from India and was considered to have acquired infection abroad. Cases 2, 3 and 4 lived in close geographical proximity in an area in the north of the city (fig 2). No other common features or exposure to potential sources of legionella were noted. It was considered that the most probable explanation for these cases was exposure to an aerosol from a cooling tower in the community as has been previously described. ${ }^{19}$ A search for cooling towers was therefore conducted in a circumscribed area of 1.5 miles radius around the place of residence of each of the three cases and including the intervening areas. Nine previously unregistered cooling towers were identified. Two were not operational, others, although satisfactory on visual inspection, required a manual dosing regimen of biocide and had no record of cleaning and maintenance since September 1992. Microbiological samples were again taken but no legionella organisms were isolated. 
Table 1 Suspected/confirmed cases of legionnaires'disease Merseyside : Fanuary/February 1995

\begin{tabular}{|c|c|c|c|c|c|c|}
\hline \multirow[b]{2}{*}{ Patient } & \multirow[b]{2}{*}{ Age } & \multirow[b]{2}{*}{ Sex } & \multicolumn{2}{|c|}{ Case category } & \multirow{2}{*}{$\begin{array}{l}\text { Risk } \\
\text { factors }\end{array}$} & \multirow[b]{2}{*}{ Evidence of other infection } \\
\hline & & & Confirmed & Presumptive & & \\
\hline 1 & $44^{\star}$ & $\mathrm{F}$ & + & & $\begin{array}{l}+ \\
(\mathrm{S})\end{array}$ & - \\
\hline 2 & 37 & M & + & & $\begin{array}{l}+ \\
(S)\end{array}$ & - \\
\hline 3 & 18 & $\mathrm{~F}$ & & + & - & $\begin{array}{l}\text { Influenza B } \\
\text { serology : CFT } 1: 20 \text { to } \\
1: 320\end{array}$ \\
\hline 4 & 27 & $\mathrm{~F}$ & & + & - & $\begin{array}{l}\text { Mycoplasma pneumoniae } \\
\text { serology : CFT <20 to } 160\end{array}$ \\
\hline 5 & 19 & M & & $\begin{array}{l}+ \\
(\S)\end{array}$ & $\begin{array}{l}+ \\
(S)\end{array}$ & $\begin{array}{l}\text { Campylobacter } \\
\text { serology positive }\end{array}$ \\
\hline 6 & 60 & $\mathrm{~F}$ & & + & $\begin{array}{l}+ \\
(\mathrm{S})(\mathrm{C})\end{array}$ & $\begin{array}{l}\text { Strep pneumoniae } \\
\text { positive blood culture }\end{array}$ \\
\hline 7 & 73 & $\mathrm{~F}$ & & + & - & $\begin{array}{l}\text { ? Q Fever } \\
\text { serology : CFT } 80 \\
C \text { burnettii } 2 \\
\text { C burnettii } \operatorname{IgG}(\mathrm{IF} / 1280)\end{array}$ \\
\hline
\end{tabular}

CFT: Complement fixation test. IF $=$ Indirect immunofluorescent antibody test. ${ }^{\star}=$ Died, $\mathrm{S}=$ smoker, $\mathrm{C}=$ chronic lung disease, $\S=$ pneumonia not confirmed by chest radiography.

\section{Microbiological investigation}

Doubts as to the validity of the Legionella pneumophila type 1 urine ELISA antigen test in this presumed case cluster were raised. Further microbiological tests indicated evidence of other infections as being responsible for the clinical findings in the presumptive cases (table 1). Two additional possible cases $(6,7)$ later identified on the basis of a positive Legionella pneumophila type 1 urine ELISA antigen test also had microbiological evidence of other infections. A limited epidemiological investigation was undertaken of the latter cases, which did not confirm any link with the previous presumed cases. The incident team considered that caution was indicated in the interpretation of the Legionella pneumophila type 1 urine ELISA antigen test in determining a need for further detailed investigation of this case cluster.

This was discussed with Central Public Health Laboratory Respiratory and Systematic Infection Laboratory, Colindale, and a laboratory error was recognised in interpretation of the appropriate "cut off" value for a positive result. This was investigated and after a careful assessment of the clinical and microbiological data, five of the seven initial positive ELISA results were reported as negative for legionella infection.

\section{Conclusion}

Despite detailed investigation of this case cluster no common source could be identified. Microbiological tests strongly suggested that five of the case cluster investigated as being legionella infections were attributable to other infections (table 1). Two cases remained on the basis of diagnostic serology for Legionella pneumophila but without any substantial epidemiological link and it was concluded that these were two sporadic cases and that the apparent cluster of seven cases constituted a pseudo outbreak of legionnaires' disease.

\section{Discussion}

Pseudo outbreaks have been previously recognised involving several other infectious diseases. ${ }^{20}{ }^{21} \mathrm{~A}$ consistent feature of such occurrences are discrepancies between clinical and epidemiological data and results of microbiological investigation. Early in the investigation of this suspected outbreak significant discrepancies were noted between the epidemiological and clinical data and the microbiological findings but it was agreed that full investigation was necessary to exclude the possibility of a common source of infection.

The age distribution of cases was noted to be atypical with some of those affected being very young for legionella infection. ${ }^{22}$ A related clinical discrepancy was the finding that few cases had recognised risk factors for legionnaires' disease. ${ }^{22}$ This suspected community outbreak also occurred in mid-winter whereas confirmed documented outbreaks of legionnaires' disease usually occur in the mid and late summer. ${ }^{23}$

The results of further microbiological investigation provide additional strong evidence for a pseudo outbreak. Confirmation of case 3 as Mycoplasma pneumoniae is consistent with the known contemporary occurrence of a community outbreak of Mycoplasma pneumoniae both nationally and on Merseyside. ${ }^{24}$ It is also consistent with reports of other members of the family suffering from upper respiratory tract infections at the time of illness. Confirmation of case 4 as influenza B infection is consistent with the history that the patient's brother had recently been admitted to hospital with pneumonia due to influenza B infection. Case 5 had recently returned from a prolonged stay in India and gave a history of diarrhoeal illness that was concluded to be attributable to campylobacter as serology indicated that seroconversion had occurred during the period abroad. It has been previously documented that cross reactions between campylobacter antibodies and diagnostic tests for legionella may occur and lead to false positive results. ${ }^{25} 26$ Furthermore, there was no conclusive evidence of pneumonia as chest radiography had not been performed.

There may be some urgency to resolve the investigation, as in this incident, where there is strong media interest about a potential source on a hospital site. A rapid microbiological test with a high predictive value is a valuable tool to guide further investigations.

It is acknowledged that, although the Legionella pneumophila type 1 urine ELISA antigen test is highly specific, the low proportion of community acquired pneumonia attributable to legionnaires' disease $(2 \%),{ }^{27}$ coupled with even a low percentage of false positives results, could significantly reduce the predictive valve of a positive test. ${ }^{13}$ When conducting investigations of suspected legionnaires' disease there remains a need for serological tests to be carried out to confirm the diagnosis and, whenever possible, legionella culture should be undertaken. ${ }^{28}$

The pseudo outbreak of legionnaires' disease we have described emphasises the importance of a strong index of suspicion when investigating suspected case clusters of legionnaires' disease. Suspicion should be aroused whenever the results of one test are not supported by others. 
We would like to thank Dr John Watson, Consultant Epidemiologist, Epidemiology Division, PHLS Communicable Disease Surveillance Centre, London, for comments on the manuscript and advice on management of the epidemiological investigation. We also wish to acknowledge the assistance of members of the Incident Team in conducting the investigation

1 First Report of the Committee of Inquiry into the Outbreak of legionnaires' disease in Stafford in April 1985. London: HMSO, 1986.

2 Fraser DW, Tsai TR, Osenstein W, et al. Legionnaires' disease. Presumption of an epidemic of pneumonia. N Engl f Med 1977;297:1189-97.

3 Marie TJ, MacDonald S, Clarlee K, et al. Nosocomial legionnaires' disease: lessons from a four year prospective study. Am 7 Infect Control 1991;19:79-85.

4 Kirby BD, Snyder KM, Meyer RD, et al. Legionnaires' disease: report of sixty-five nosocomially acquired cases and review of the literature. Medicine (Baltimore) 1980;59: $188-205$.

5 Fallon RJ, Reid D, Donaldson JR, et al. Outbreak of legionellosis in a community. Lancet 1986 ;ii:380-3.

6 Addiss DG, David JP, LaVenture M. Community acquired Addiss DG, David JP, LaVenture M. Community acquired legionnaires' disease associated with a cooling tower: mophila. Am 7 Epidemiol 1989;130:557-68.

7 Westminster Action Committee. Broadcasting House legionnaires'disease. London: City of Westminster Environmental naires'disease. London: City

8 Sivann RA, Peel MM, Rouch GJ. Isolation of Legionella pneumophila from hospital water systems in Victoria. Med $\mathcal{F}$ Aust 1982;2:226-7.

9 Fleismans CB, Cherry WB, Orrison LH, et al. Isolation of Legionella pneumophila from non-epidemic related aquatic habitats. Appl Environ Microbiol 1979;37:1239-42.

10 Saunders CJP, Joseph CA, Watson JM. Investigating a single case of legionnaires' disease: guidance for consultants in communicable disease control. Commun Dis Rep 1994;4: R112-14.

11 Kohler RB. Antigen detection for the rapid diagnosis of Mycoplasma and Legionella pneumonia. Diagn Microbiol Mycoplasma and Legionella pneumo
Infect Dis 1986;4 (suppl 3):475-595.

12 Kohler RB. Legionella antigenuria: testing and interpretation. Clin Microbiol Newsletter 1990;12:185-8.
13 Birtles RI, Harrison TG, et al. Evaluation of a urinary antien ELISA for the diagnosis of Legionella pneumophila serogen ELISA for the diagnosis of Legionella pneumophi
group 1 infection. $\mathcal{F}$ Clin Pathol 1990;43:685-90.

14 Harrison TG, Taylor AG. A rapid microagglutination test for the diagnosis of Legionella pneumophila (serogroup 1) infections. F Clin Pathol 1982;35:1028-31.

15 Lyon D. The rapid microagglutination test in the diagnosis of legionnaires' disease. Serodiagnosis Immunotherapy 1987; 1:79-82.

16 Wilkinson HW, et al. Indirect IFA test for legionnaires' disease in "legionnaires: the disease, bacterium and methodology". In: Jones GL, ed. Atlanta: US Department of Health Education and Welfare, 1978:111-16.

17 NHS Estates Health Technical Memorandum 2040. The control of Legionella in health care premises - a code of practice. London: HMSO, 1993.

18 England AC, Fraser DW, Plikaytis BD, et al. Sporadic legionellosis in the United States; the first thousand cases. Ann Intern Med 1981;94:164-70.

9 Bhopal R. Source of infection of sporadic legionnaires' disease: a review. F Infect 1995;30:9-12.

20 Shears P, Rhodes LE, Syed Q, et al. A pseudo outbreak of tuberculosis. Commun Dis Rev 1994;4:R9-10.

21 Weinstein RA, Stam WE. Pseudo-epidemics in hospitals. Lancet 1977;ii:862-4.

22 Yu VL. Legionella pneumophila C legionnaires' disease. In: Mandell GL, Bennett JE, Dolin R, eds. Principles and practice of infectious disease. New York: Churchill Livingstone, 1993:2089.

23 Anonymous. Legionnaires' disease in England and Wales 1997. Commun Dis Rep 1997;36:321, 324

24 Anonymous. Current respiratory infections. Commun Dis Rep 1995;5:21.

25 Cheesebrough J, Makin T, Taxman B, et al. False-positive Legionella serology in Campylobacter infection. Lancet 1992;339:429.

26 Boswell TCJ, Marshall LE, Kudesia G. False-positive Legionella titres in routine clinical serology testing detected by absorption with Campylobacter: implications for the serological diagnosis of legionnaires' disease. F Infect 1996; $32:: 23-6$.

27 Reingold AL. Role of Legionellae in acute infections of the lower respiratory tract. Rev Infect Dis 1988;10:1018-28.

28 Edelstein PH. Legionnaires' disease. Clin Infect Dis 1983;16: 741-9. 